Teresa H. Chu

\title{
A class of polynomially solvable linear complementarity problems
}

Received: August 5, 2002 / Accepted: September 6, 2005

Published online: December 6, 2005 - (C) Springer-Verlag 2005

\begin{abstract}
Although the general linear complementarity problem (LCP) is NP-complete, there are special classes that can be solved in polynomial time. One example is the type where the defining matrix is nondegenerate and for which the $n$-step property holds. In this paper we consider an extension of the property to the degenerate case by introducing the concept of an extended $n$-step vector and matrix. It is shown that the LCP defined by such a matrix is polynomially solvable as well.
\end{abstract}

\section{Introduction}

Given a real square matrix $M$ of order $n$ and a vector $q \in \mathbb{R}^{n}$, the linear complementarity problem, denoted by $\operatorname{LCP}(q, M)$, is that of finding $z, w \in \mathbb{R}^{n}$ such that

$$
w=M z+q, z \geq 0, w \geq 0, \text { and } z^{\mathrm{T}} w=0 .
$$

Although the general problem (with arbitrary data $M$ and $q$ ) is NP-complete in the strong sense [3], there are known classes that can be processed efficiently. (Processing a problem means finding a solution or concluding definitively the nonexistence of one.) These include, for example, the cases where the given matrix $M$ is positive semidefinite [9], has nonpositive off-diagonal entries (see [1], [17], and [11]), or has a certain " $n$-step" property [14].

Generally speaking, the computational complexity of an algorithm depends not only on the matrix properties of $M$ but also on the vector $q$ furnished by the data. In this paper we study a class of problems for which a solution can always be computed in polynomial time for any $q$, and for which $q$ plays no significant role in the computational complexity. This class is an extension of the one presented by Pang and Chandrasekaran [14]. The characterization of the problems considered in [14] involves what is known as an $n$-step vector, where it has been observed that by using this type of vector, a family of LCPs can be solved by two well-known algorithms in no more than $n$ iterations, hence the name " $n$-step." For the $n$-step property to hold, the given matrix $M$ is assumed to be at least nondegenerate; that is to say, all principal submatrices of $M$ must be nonsingular. The major part of this work is the extension of these algorithmic results to the degenerate case by enlarging the definition of the $n$-step vector to cover this case.

The paper is organized as follows. In the next section we present essential background material, including two of the main results from [14]. In Section 3 we define the

T. H. Chu: Dept. of Ind. \& Oper. Eng., University of Michigan, Ann Arbor, MI 48109, USA. e-mail: tchu@umich. edu 
extended $n$-step (ENS) vector and the corresponding matrix by giving two equivalent definitions. The two results of [14] are then extended using the more general definitions; these are proved separately in Sections 4 and 5.

\section{Background}

\subsection{Miscellaneous preliminaries}

We begin by briefly explaining the notation. Let us denote the space of real $n \times n$ matrices by $\mathbb{R}^{n \times n}$, the real $n$-space by the usual $\mathbb{R}^{n}$ symbol, and the nonnegative (positive) orthant of $\mathbb{R}^{n}$ by $\mathbb{R}_{+}^{n}\left(\mathbb{R}_{++}^{n}\right)$. For an index subset $\alpha \subseteq\{1, \ldots, n\}, \bar{\alpha}=\{1, \ldots, n\} \backslash \alpha$, and its cardinality is indicated by the symbol $|\alpha|$. The $i$-th row of $A \in \mathbb{R}^{n \times n}$ is denoted by $A_{i}$. and its $j$-th column by $A$. $j$. For $\alpha, \beta \subseteq\{1, \ldots, n\}, A_{\alpha \beta}$ is the submatrix of $A$ obtained by deleting all rows and columns indexed by $\bar{\alpha}$ and $\bar{\beta}$, respectively. In the case where $\alpha=\beta, A_{\alpha \alpha}$ is called the principal submatrix of $A$ determined by $\alpha$. The polyhedral cone pos $A$ is the set $\left\{y \in \mathbb{R}^{n}: y=A x\right.$ for some $\left.x \in \mathbb{R}_{+}^{n}\right\}$, consisting of all nonnegative linear combinations of the columns of $A$. Lastly, a line over a matrix class represents the property of completeness - a matrix belongs to a particular complete class if and only if all its principal submatrices belong to the same class as well.

We have already identified a nondegenerate matrix as one that has no zero principal minor; it is degenerate otherwise. Additional classes that are relevant to our subject are listed below, where a bold capital letter is used to describe a matrix satisfying the definition listed next to it, as well as to represent the collection of such objects. All matrices are taken to be real and square. (See Murty [12] and Cottle, Pang, and Stone [7] for discussions on matrix classes.)

$\boldsymbol{P}:\left(\boldsymbol{P}_{\mathbf{0}}\right): \quad$ A matrix whose principal minors are all positive (nonnegative).

$Q: \quad \quad$ A matrix $M$ for which the $\operatorname{LCP}(q, M)$ has a solution for every $q \in \mathbb{R}^{n}$.

$S: \quad$ A matrix $M$ for which there exists a positive vector $x$ such that $M x>0$.

$Z$ : $\quad$ A matrix whose off-diagonal entries are all nonpositive.

$\boldsymbol{K}:\left(\boldsymbol{K}_{\mathbf{0}}\right): \quad$ The intersection of the classes $\boldsymbol{P}\left(\boldsymbol{P}_{\mathbf{0}}\right)$ and $\boldsymbol{Z}$. The $\boldsymbol{K}$-matrices are also called Minkowski matrices.

hidden $Z$ : $\quad$ A matrix $M$ for which there exist $Z$-matrices $X$ and $Y$ such that $M X=$ $Y$ and $r^{\mathrm{T}} X+s^{\mathrm{T}} Y>0$ for some nonnegative vectors $r$ and $s$.

The following characterizations will be useful.

Theorem 1 ([5],[16]). The classes $\overline{\boldsymbol{Q}}$ and $\overline{\boldsymbol{S}}$ are identical.

Theorem 2 ([13]). Suppose $M \in \mathbb{R}^{n \times n}$ is a hidden Z-matrix. Then $M \in \boldsymbol{P}$ iff $M \in \overline{\boldsymbol{S}}$.

\section{Schur Complements and Principal Pivot Transforms}

Let $M \in \mathbb{R}^{n \times n}$ be partitioned into $M=\left[\begin{array}{ll}M_{\alpha \alpha} & M_{\alpha \bar{\alpha}} \\ M_{\bar{\alpha} \alpha} & M_{\bar{\alpha} \bar{\alpha}}\end{array}\right]$, where $M_{\alpha \alpha}$ is a nonsingular submatrix of $M$. The Schur complement of $M$ with respect to $\alpha$, denoted $\left(M / M_{\alpha \alpha}\right)$, is equal to this square matrix: $M_{\bar{\alpha} \bar{\alpha}}-M_{\bar{\alpha} \alpha}\left(M_{\alpha \alpha}\right)^{-1} M_{\alpha \bar{\alpha}}$.

The next proposition implies that a square matrix is singular if and only if a Schur complement of it is singular. A proof can be found in [7]. 
Proposition 3. Let $M \in \mathbb{R}^{n \times n}$ be partitioned into

$$
M=\left[\begin{array}{ll}
M_{\alpha \alpha} & M_{\alpha \bar{\alpha}} \\
M_{\bar{\alpha} \alpha} & M_{\bar{\alpha} \bar{\alpha}}
\end{array}\right],
$$

where $\alpha=\{1, \ldots, r\}$ and $r<n$. If $M_{\alpha \alpha}$ is invertible, then

$$
\operatorname{det} M / \operatorname{det} M_{\alpha \alpha}=\operatorname{det}\left(M / M_{\alpha \alpha}\right) \text {. }
$$

If, in addition, $\left(M / M_{\alpha \alpha}\right)$ is invertible, then $M^{-1}=$

$$
\left[\begin{array}{c}
\left(M_{\alpha \alpha}\right)^{-1}+\left(M_{\alpha \alpha}\right)^{-1} M_{\alpha \bar{\alpha}}\left(M / M_{\alpha \alpha}\right)^{-1} M_{\bar{\alpha} \alpha}\left(M_{\alpha \alpha}\right)^{-1}-\left(M_{\alpha \alpha}\right)^{-1} M_{\alpha \bar{\alpha}}\left(M / M_{\alpha \alpha}\right)^{-1} \\
-\left(M / M_{\alpha \alpha}\right)^{-1} M_{\bar{\alpha} \alpha}\left(M_{\alpha \alpha}\right)^{-1}
\end{array}\right] .
$$

Let $P$ be a permutation matrix and $M$ a square matrix. Then $P M P^{\mathrm{T}}$ is called a principal rearrangement of $M$. Let $M \in \mathbb{R}^{n \times n}$ and $\alpha \subseteq\{1, \ldots, n\}$ with $\operatorname{det} M_{\alpha \alpha} \neq 0$. Then, up to principal rearrangement, the principal pivot transform $(P P T)$ of $M$ with respect to $\alpha$ is given by the matrix

$$
\left[\begin{array}{cc}
\left(M_{\alpha \alpha}\right)^{-1} & -\left(M_{\alpha \alpha}\right)^{-1} M_{\alpha \bar{\alpha}} \\
M_{\bar{\alpha} \alpha}\left(M_{\alpha \alpha}\right)^{-1} & \left(M / M_{\alpha \alpha}\right)
\end{array}\right] .
$$

\subsection{The n-step property: nondegenerate case}

In this section we present the main algorithmic results for nondegenerate-matrix LCPs as set forth in [14]. The nondegenerate case provides the framework and motivation for the work to follow.

Definition 4. Let $M \in \mathbb{R}^{n \times n}$ be nondegenerate and $p$ a positive vector in $\mathbb{R}^{n}$. Then $p$ is called an $n$-step vector for $M$ if

$$
\left(M_{\alpha \alpha}\right)^{-1} p_{\alpha}>0 \quad \forall \alpha \subseteq\{1, \ldots, n\} .
$$

The notion of " $n$-step" apparently was based on the results in [14], but it was not named as such in that paper. As a matter of fact, the vector $p$ there was defined in a slightly different manner, as a positive vector $p$ satisfying the condition

$$
\left(M_{\alpha \alpha}\right)^{-1} p_{\alpha} \geq 0 \quad \forall \alpha \subseteq\{1, \ldots, n\}
$$

rather than the strict inequalities in (1). (Condition (2) also appeared in [4].) In Definition 4 , however, we have adopted the terminology subsequently employed in [7]. An interesting observation is that the set of positive vectors satisfying (2) may be different from the closure of the set of vectors satisfying (1), even for a $\boldsymbol{P}$-matrix. (A numerical example illustrating this fact is given in [2].)

Theorem 5 ([14]). Let $M \in \mathbb{R}^{n \times n}$ be a P-matrix and $q \in \mathbb{R}^{n}$ be arbitrary. Suppose there exists a positive vector p satisfying inequalities (2). Then the parametric principal pivoting algorithm [4], using $p$ as the parametric vector, will compute a solution to $\operatorname{LCP}(q, M)$ in at most $n$ pivot steps. 
Theorem 6 ([14]). Let $M \in \mathbb{R}^{n \times n}$ be nondegenerate and $q \in \mathbb{R}^{n}$ be arbitrary. Suppose there exists a positive vector p satisfying inequalities (2). Then Lemke's algorithm [10], using $p$ as the covering vector, will terminate with a solution to $\operatorname{LCP}(q, M)$ after at most $n+1$ pivot steps.

The above algorithms refer to two known techniques (to the LCP community) that will be described briefly in Sections 4 and 5. With this background material in place, we are now in a position to establish the new results.

\section{The extended property}

In this section we introduce the extended $n$-step property by presenting two equivalent definitions.

Definition 7. Let $M \in \mathbb{R}^{n \times n}$ and $p \in \mathbb{R}_{++}^{n}$ be given. The vector $p$ is called an extended $n$-step (abbreviated ENS) vector for $M$ if both conditions below are satisfied:

(i) $\left(M_{\alpha \alpha}\right)^{-1} p_{\alpha} \geq 0 \quad \forall M_{\alpha \alpha}$ nonsingular

(ii) $\operatorname{rank}\left(M_{\alpha \alpha} \mid p_{\alpha}\right)<|\alpha| \quad \forall M_{\alpha \alpha}$ singular

where $\left(M_{\alpha \alpha} \mid p_{\alpha}\right)$ is an augmented matrix. The set of ENS vectors for $M$ will be denoted by $\boldsymbol{\Delta}_{M}$. The matrix $M$ is said to be an ENS matrix if $\boldsymbol{\Delta}_{M}$ is nonempty. Conditions ( $i$ ) and (ii) together shall be referred to as the ENS property (of $M$ ) if such a vector p exists.

If the given matrix is nondegenerate, then the definition reduces to that of a vector satisfying Equation (2). Notice also that any ENS matrix must necessarily have positive diagonal elements because the associated vector is positive.

There is a more succinct, alternative way of characterizing the extended property that can be expressed in terms of finitely generated cones.

Theorem 8. Let $M \in \mathbb{R}^{n \times n}$ and $p \in \mathbb{R}_{++}^{n}$ be given. Then $p$ is an extended $n$-step vector for $M$ if and only if $p_{\alpha} \in \operatorname{pos} M_{\alpha \alpha}$ for all $\alpha \subseteq\{1, \ldots, n\}$.

Proof. That the latter implies the former is clear. To prove the converse, suppose $p$ is an extended $n$-step vector. We must show that $p_{\alpha} \in \operatorname{pos} M_{\alpha \alpha}$ for every $\alpha$. The proof is by induction on $r=|\alpha|$. The case where $r=1$ is trivially true because $M$ has positive diagonal elements. Now suppose $r>1$. If $M_{\alpha \alpha}$ is nonsingular, then $\left(M_{\alpha \alpha}\right)^{-1} p_{\alpha} \geq 0$ by condition (i) of Definition 7 and $p_{\alpha} \in \operatorname{pos} M_{\alpha \alpha}$. If $M_{\alpha \alpha}$ is singular, by (ii) of Definition 7, $\left(M_{\alpha \alpha} \mid p_{\alpha}\right)$ has a row, say the $\alpha_{k}$-th row, that is a linear combination of the other rows. Delete this row from $\left(M_{\alpha \alpha} \mid p_{\alpha}\right)$ and consider the reduced system

$$
\left[\begin{array}{ccc}
m_{\alpha_{1} \alpha_{1}} & \cdots & m_{\alpha_{1} \alpha_{r}} \\
\vdots & \cdots & \vdots \\
m_{\alpha_{k-1}, \alpha_{1}} & \cdots & m_{\alpha_{k-1}, \alpha_{r}} \\
m_{\alpha_{k+1}, \alpha_{1}} & \cdots & m_{\alpha_{k+1}, \alpha_{r}} \\
\vdots & \cdots & \vdots \\
m_{\alpha_{r} \alpha_{1}} & \cdots & m_{\alpha_{r} \alpha_{r}}
\end{array}\right] x_{\alpha}=\left[\begin{array}{c}
p_{\alpha_{1}} \\
\vdots \\
p_{\alpha_{k-1}} \\
p_{\alpha_{k+1}} \\
\vdots \\
p_{\alpha_{r}}
\end{array}\right] .
$$


It is equivalent to the undeleted one. Set $\beta=\left\{\alpha_{1}, \ldots, \alpha_{r}\right\} \backslash\left\{\alpha_{k}\right\}$. The pair $\left\{M_{\beta \beta}\right.$, $\left.p_{\beta}\right\}$ satisfies the ENS property. So by the induction hypothesis there is a solution $\left(\bar{x}_{1}, \ldots, \bar{x}_{k-1}, \bar{x}_{k+1}, \ldots, \bar{x}_{r}\right)^{\mathrm{T}}$ to $M_{\beta \beta} x_{\beta}=p_{\beta}$ that is nonnegative. Set $\bar{x}_{\alpha}=\left(\bar{x}_{1}, \ldots\right.$, $\left.\bar{x}_{k-1}, 0, \bar{x}_{k+1}, \ldots, \bar{x}_{r}\right)^{\mathrm{T}}$. Then $\bar{x}_{\alpha} \geq 0$ solves (3) and, therefore, the original system $M_{\alpha \alpha} x_{\alpha}=p_{\alpha}$ as well.

Remark 9. A more restrictive set of conditions, $p_{\alpha} \in \operatorname{int} \operatorname{pos} M_{\alpha \alpha}$ for all $\alpha \subseteq\{1, \ldots, n\}$, appeared in [8] as a generalization of the properties of being a centrally projected point or an $n$-step vector. (CP points for a simplicial cone defined by a nonsingular matrix were shown in that paper to be in 1-1 correspondence with the $n$-step vectors for a related matrix.)

\section{Linear termination of Lemke's method}

One of the oldest and perhaps the most widely known methods for solving linear complementarity problems is the complementary pivoting algorithm due to Lemke [10]. The following version, taken from [7], is presented in a slightly different form.

\section{Algorithm 10.}

Step 0. (Initialization.) If $q \geq 0$, terminate; $z=0$ solves $\operatorname{LCP}(q, M)$. Otherwise, introduce the artificial variable $z_{0}$ and a covering vector $p>0$ associated with it. Consider the augmented problem:

$$
\begin{aligned}
& w-M z-p z_{0}=q \\
& w \geq 0, z \geq 0, z_{0} \geq 0
\end{aligned}
$$

Let $r=\arg \max \left\{\left|q_{i}\right| / p_{i}: q_{i}<0\right\}$. Pivot on the element at position $\left(w_{r}, z_{0}\right)$. Now both $w_{r}$ and $z_{r}$ are nonbasic variables. The initial basic feasible vector to this system is $\left(w_{1}, \ldots, w_{r-1}, z_{0}, w_{r+1}, \ldots, w_{n}\right)$. Choose the driving variable to be $z_{r}$.

Step 1. (Determination of the blocking variable, if any.) If the column of the driving variable has at least one positive entry, perform a (lexico) minimum ratio test to determine the basic variable that blocks the increase of the driving variable. If the driving variable is unblocked, then it can be increased arbitrarily, and secondary ray termination occurs. Terminate; the method has failed to find a solution.

Step 2. (Pivoting.) The blocking variable is known. If it is $z_{0}$, then pivot on the element at position $\left(z_{0}\right.$, driving variable) and terminate. A solution is obtained by the current basic feasible solution. Otherwise, pivot on the element located in the row of the blocking variable and the column of the driving variable. Go to Step 1 using the complement of the current blocking variable as the new driving variable.

Remark 11. The problem of cycling under degeneracy of a pivot step (i.e., one of zero length) in Lemke's method is resolved by adopting the lexico minimum rule (in Step 1) to identify the blocking variable uniquely in every iteration. For the $n$-step procedures discussed here, however, linear termination holds even under degeneracy without any need of a special minimum rule. 
When applied to a $\boldsymbol{P}$-matrix or any $\overline{\boldsymbol{Q}}$-matrix problem, this method will compute a solution using any positive covering vector [6], but the number of iterations required may be exponential. (See [12] for numerical examples that exhibit this worst-case behavior.) Theorem 6, however, ensures against the possibility of an undesirable termination rate if the matrix $M$ is nondegenerate and possesses an $n$-step vector that can be used to initiate the algorithm.

In view of the ENS property defined in the preceding section, Theorem 6 can now be extended accordingly to accommodate the degenerate case. The gist of the argument used in both cases is that once a $z$-variable becomes basic, it cannot be dropped from the basic vector in subsequent iterations.

Theorem 12. Let $M \in \mathbb{R}^{n \times n}$ be an ENS matrix, $p \in \boldsymbol{\Delta}_{M}$, and $q \in \mathbb{R}^{n}$ be arbitrary. Using $p$ as the covering vector, Lemke's algorithm will terminate with a solution to the $\operatorname{LCP}(q, M)$ after at most rank $(M)$ iterations.

Proof. The initial tableau of the algorithm is:

\begin{tabular}{lllll}
\hline b.v. & $w$ & $z$ & $z_{0}$ & \\
\hline$w_{1}$ & & & $-p_{1}$ & $=q_{1}$ \\
$\vdots$ & $I_{n \times n}$ & $-M$ & $\vdots$ & $\vdots$ \\
$w_{n}$ & & & $-p_{n}$ & $=q_{n}$ \\
\hline
\end{tabular}

If $q \geq 0$, then $z=0$ solves $\operatorname{LCP}(q, M)$. So assume $q$ has a negative component. Suppose at the beginning of the first iteration after initialization $z_{0}$ has become basic and $w_{r}$ nonbasic, so that $z_{r}$ is the driving variable. The $\left(z_{0}, z_{r}\right)$ entry is $m_{r r} / p_{r}$ where $p_{r}>0$. Since $M$ has positive diagonal elements, $m_{r r}>0$. Termination on a secondary ray thus cannot occur at this point.

Consider an iteration (after the first) where the pair $\left\{z_{t}, w_{t}\right\}$ is nonbasic, with $w_{t}$ having left the basis in the previous iteration. The driving variable is $z_{t}$. The current tableau may be written as:

\begin{tabular}{ll}
\hline b.v. & $z_{t}$ \\
\hline$z_{\alpha}$ & $\left(\begin{array}{ll}M_{\alpha \alpha} & p_{\alpha} \\
M_{t \alpha} & p_{t}\end{array}\right)^{-1}\left(\begin{array}{c}M_{\alpha t} \\
M_{t t}\end{array}\right)$ \\
$z_{0}$ & \\
\hline$w_{\beta}$ & \\
\hline
\end{tabular}

Here, only the relevant entries $\left(z_{\alpha}, z_{t}\right)$ and $\left(z_{0}, z_{t}\right)$ are displayed. The set $\alpha$ is the index set corresponding to the basic $z$ variables, $t$ is the index of the nonbasic pair $\left\{z_{t}, w_{t}\right\}$, and $\beta$ is the complement of $\alpha \cup\{t\}$.

Now, the matrix $A=\left[\begin{array}{cc}M_{\alpha \alpha} & p_{\alpha} \\ M_{t \alpha} & p_{t}\end{array}\right]$ is nonsingular because $\left(z_{\alpha}, z_{0}, w_{\beta}\right)$ is a basic vector. This means the principal submatrix $M_{\bar{\beta} \bar{\beta}}=\left[\begin{array}{cc}M_{\alpha \alpha} & M_{\alpha t} \\ M_{t \alpha} & M_{t t}\end{array}\right]$ is nonsingular because, otherwise, by condition (ii) of Definition 7, the rows of $\left(M_{\bar{\beta} \bar{\beta}} \mid p_{\bar{\beta}}\right)$ would be linearly dependent, and $A$ would be singular. By condition (i) of the same definition then,

$$
\left[\begin{array}{c}
\tilde{p}_{\alpha} \\
\tilde{p}_{t}
\end{array}\right]=\left[\begin{array}{cc}
M_{\alpha \alpha} & M_{\alpha t} \\
M_{t \alpha} & M_{t t}
\end{array}\right]^{-1}\left[\begin{array}{c}
p_{\alpha} \\
p_{t}
\end{array}\right] \geq 0 .
$$


The vector

$$
\left[\begin{array}{c}
\left(z_{\alpha}, z_{t}\right) \text { entry } \\
\left(z_{0}, z_{t}\right) \text { entry }
\end{array}\right]=\left[\begin{array}{cc}
M_{\alpha \alpha} & p_{\alpha} \\
M_{t \alpha} & p_{t}
\end{array}\right]^{-1}\left[\begin{array}{c}
M_{\alpha t} \\
M_{t t}
\end{array}\right]
$$

By Cramer's rule, $\tilde{p}_{t}=\frac{\operatorname{det} A}{\operatorname{det} M_{\bar{\beta} \bar{\beta}}}>0$, and the $\left(z_{0}, z_{t}\right)$ entry is the positive number $1 / \tilde{p}_{t}$, so that ray termination cannot occur. From Equations (4) and (5) it is easy to see that the $\left(z_{\alpha}, z_{t}\right)$ entry is equal to $-\tilde{p}_{\alpha} / \tilde{p}_{t}$, which is nonpositive. Hence, the blocking variable will not be chosen from the components of $z_{\alpha}$. Since the above argument shows that a $z$-variable that has become basic cannot become nonbasic again, the algorithm will terminate after at $\operatorname{most} \max \left\{|\alpha|:\left(M_{\alpha \alpha}\right)^{-1}\right.$ exists $\} \leq \operatorname{rank}(M)$ iterations with a solution.

As in [14] no nondegeneracy assumption of the pivot steps is required in the proof. Notice also that since each pivot step requires at most $\mathrm{O}\left(n^{2}\right)$ arithmetic operations, the result tells us that the LCP in question can be solved in $\mathrm{O}\left(n^{3}\right)$ time.

Corollary 13. Extended $n$-step matrices belong to $\overline{\boldsymbol{Q}}$, or equivalently, to $\overline{\boldsymbol{S}}$.

Proof. Since the ENS property is clearly inheritable by all its principal submatrices, the conclusion follows from Theorems 12 and 1.

Corollary 14. Suppose a matrix $M \in \mathbb{R}^{n \times n}$ has a nonempty $\Delta_{M}$ set. Then the following statements hold.

(i) If $M$ is $\boldsymbol{Z}$, then $M \in \boldsymbol{P}$.

(ii) If $M$ is hidden $\boldsymbol{Z}$, then $M \in \boldsymbol{P}$.

Proof. It suffices to prove statement (ii). Since $\boldsymbol{\Delta}_{M}$ is nonempty, $M$ belongs to $\bar{S}$ (Corollary 13). It follows from Theorem 2 that $M \in \boldsymbol{P}$.

Corollary 15. Let $M \in \mathbb{R}^{n \times n} \cap\left(\boldsymbol{P}_{\mathbf{0}} \backslash \boldsymbol{P}\right)$ be a hidden $\boldsymbol{Z}$-matrix. Then $\boldsymbol{\Delta}_{M}=\emptyset$. In particular, a $\left(\boldsymbol{K}_{\mathbf{0}} \backslash \boldsymbol{K}\right)$-matrix has no extended n-step vector.

\section{Linear termination of parametric LCP algorithm with positive parametric vector}

The goal of this section is to prove a more general version of Theorem 5 . We first define the parametric LCP.

Suppose $M \in \mathbb{R}^{n \times n}$ and $p, q \in \mathbb{R}^{n}$ where $p$ is nonzero. The parametric linear complementarity problem $\operatorname{PLCP}(q, p, M)$ is to find, for each $\lambda$ in some interval $\left[\lambda_{*}, \infty\right)$, a $z \in \mathbb{R}^{n}$ — which may depend on $\lambda$-satisfying:

$$
w=q+\lambda p+M z \geq 0, \quad z \geq 0, \quad \text { and } \quad z^{\mathrm{T}} w=0 .
$$

In this problem, the vector $p$ is called the parametric vector associated with the real parameter $\lambda$. In what follows we shall assume that the given parametric vector is positive. 
A general parametric principal pivoting algorithm (PPPA) has been developed in [4] to process specifically those PLCPs for which the pivot element is either positive or zero at each iteration. (If a negative pivot is encountered, then the algorithm is unable to proceed, and it terminates.) In the former case, a single principal pivot step is performed; in the latter a double principal pivot (or block pivot) step is performed. At each iteration of the PPPA, the updated current tableau contains the transforms of the original data $-q$, $p$, and $M$. The pivot element is selected from among the diagonal entries of the current PPT of $M$ in the tableau based on a ratio test, comparing only those rows $i$ for which the current $p_{i}$-th element is positive. If the updated $p$ column consists of only nonpositive entries, then the algorithm terminates with a solution function $z(\lambda)$ for all $\lambda$ in the desired range. This is another way in which the algorithm can terminate. If for some $\lambda$ in the search interval $\left[\lambda_{*}, \infty\right)$ the PLCP has no solution, then we have met a third termination criterion. These are all the possible outcomes of the general PPPA.

Developed in [15], the algorithm we are about to present is a version of the PPPA that can be used only if all pivot elements are known to be positive; such is the case for a $\boldsymbol{P}$-matrix, for example. In this version, a tie-breaking rule suggested in [12] for determining the maximizing index $r$ has been incorporated into Step 2.

\section{Algorithm 16. Parametric principal pivoting algorithm using only single principal pivot steps}

Step 0. (Initialization.) Let $\lambda_{\text {old }}=\infty, \alpha=\emptyset$, and $\bar{\alpha}=\{1, \ldots, n\}$.

Step 1. Compute: $\left(\tilde{q}_{\alpha}, \tilde{p}_{\alpha}\right)=-\left(M_{\alpha \alpha}\right)^{-1}\left(q_{\alpha}, p_{\alpha}\right)$ and $\left(\tilde{q}_{\bar{\alpha}}, \tilde{p}_{\bar{\alpha}}\right)=\left(q_{\bar{\alpha}}, p_{\bar{\alpha}}\right)+$ $M_{\bar{\alpha} \alpha}\left(\tilde{q}_{\alpha}, \tilde{p}_{\alpha}\right)$.

Step 2. If $\tilde{p} \leq 0$, set $(z(\lambda))_{\bar{\alpha}}=0$ and $(z(\lambda))_{\alpha}=-\tilde{q}_{\alpha}-\lambda \tilde{p}_{\alpha}$, for each $\lambda \in$ $\left(-\infty, \lambda_{\text {old }}\right]$. Terminate. Otherwise, perform the ratio test $\lambda_{\text {new }}=\max _{i}$ $\left\{-\tilde{q}_{i} / \tilde{p}_{i}: \tilde{p}_{i}>0\right\}$, and let $r=\max \left\{\arg \max _{i}\left\{-\tilde{q}_{i} / \tilde{p}_{i}: \tilde{p}_{i}>0\right\}\right\}$. Set $(z(\lambda))_{\bar{\alpha}}=0$ and $(z(\lambda))_{\alpha}=-\tilde{q}_{\alpha}-\lambda \tilde{p}_{\alpha} \forall \lambda \in\left[\lambda_{\text {new }}, \lambda_{\text {old }}\right]$. If $\lambda_{\text {new }} \leq \lambda_{*}$, terminate. Otherwise, set $\lambda_{\text {old }}=\lambda_{\text {new }}$.

Step 3. Update index sets $\alpha$ and $\bar{\alpha}$ :

$$
\alpha_{\text {new }}= \begin{cases}\alpha_{\text {old }} \cup r & \text { if } r \notin \alpha_{\text {old }} \\ \alpha_{\text {old }} \backslash r & \text { if } r \in \alpha_{\text {old }}\end{cases}
$$

Set $\bar{\alpha}_{\text {new }}=\{1, \ldots, n\} \backslash \alpha_{\text {new }}$ and go to Step 1 .

Theorem 17. Suppose $M \in \mathbb{R}^{n \times n}$ is an ENS matrix whose PPTs have only nonnegative elements on the main diagonal. Let $p, q \in \mathbb{R}^{n}$ with $p>0$. If $p \in \boldsymbol{\Delta}_{M}$, then the parametric principal pivoting algorithm will find a solution function $z(\lambda), \lambda \in \mathbb{R}$, to the problem $\operatorname{PLCP}(q, p, M)$ in at most $\operatorname{rank}(M)$ iterations.

Proof. The initial canonical system in tableau form is

\begin{tabular}{llll}
\hline b.v. & & $\lambda$ & $z$ \\
\hline$w_{1}=$ & $q_{1}$ & $p_{1}$ & \\
$\vdots$ & $\vdots$ & $\vdots$ & $M$ \\
$w_{n}=$ & $q_{n}$ & $p_{n}$ & \\
\hline
\end{tabular}


Based on results in the previous section (Corollary 13) we know that ENS matrices belong to the class $\overline{\boldsymbol{Q}}$. Therefore, the parametric LCP under consideration has a solution for each $\lambda \in \mathbb{R}$. By hypothesis, the diagonal entries of every PPT of $M$ are nonnegative. We now show that the pivot in every iteration of the (general) PPP algorithm is not only nonnegative but is in fact positive; hence, Algorithm 16 may be used.

Since all the diagonal entries of $M$ are positive, the first pivot step is simple; that is, no double principal pivot step is required. Now suppose we are at some iteration after the first, where the canonical tableau is

\begin{tabular}{lllll}
\hline b.v. & & $\lambda$ & $w_{\alpha}$ & $z_{\bar{\alpha}}$ \\
\hline$z_{\alpha}=$ & $\tilde{q}_{\alpha}$ & $\tilde{p}_{\alpha}$ & $\left(M_{\alpha \alpha}\right)^{-1}$ & $-\left(M_{\alpha \alpha}\right)^{-1} M_{\alpha \bar{\alpha}}$ \\
$w_{\bar{\alpha}}=$ & $\tilde{q}_{\bar{\alpha}}$ & $\tilde{p}_{\bar{\alpha}}$ & $M_{\bar{\alpha} \alpha}\left(M_{\alpha \alpha}\right)^{-1}$ & $\left(M / M_{\alpha \alpha}\right)$ \\
\hline
\end{tabular}

At this stage $\alpha$ and $\bar{\alpha}$ are the index sets of the basic $z$ and $w$-variables, respectively. The transformed $p$ and $q$ corresponding to the current PPT of $M$ are

$$
\begin{aligned}
& \left(\tilde{q}_{\alpha}, \tilde{p}_{\alpha}\right)=-\left(M_{\alpha \alpha}\right)^{-1}\left(q_{\alpha}, p_{\alpha}\right) \\
& \left(\tilde{q}_{\bar{\alpha}}, \tilde{p}_{\bar{\alpha}}\right)=\left(q_{\bar{\alpha}}, p_{\bar{\alpha}}\right)+M_{\bar{\alpha} \alpha}\left(\tilde{q}_{\alpha}, \tilde{p}_{\alpha}\right) .
\end{aligned}
$$

The next pivot $m_{r r}^{\prime}$ is chosen from the main diagonal of the principal pivot transform (w.r.t. $\alpha$ ) given in the above tableau. Since $\tilde{p}_{\alpha}=-\left(M_{\alpha \alpha}\right)^{-1} p_{\alpha} \leq 0$ by hypothesis, $r$ cannot be contained in $\alpha$. So the pivot must be selected from among the diagonal entries of the Schur complement $\left(M / M_{\alpha \alpha}\right)=M_{\bar{\alpha} \bar{\alpha}}-M_{\bar{\alpha} \alpha}\left(M_{\alpha \alpha}\right)^{-1} M_{\alpha \bar{\alpha}}$. Suppose $\left(M / M_{\alpha \alpha}\right)$ has a $k$-th diagonal element that is zero. This means the matrix

$$
\left[\begin{array}{cc}
M_{\alpha \alpha} & M_{\alpha \bar{\alpha}_{k}} \\
M_{\bar{\alpha}_{k} \alpha} & M_{\bar{\alpha}_{k} \bar{\alpha}_{k}}
\end{array}\right]
$$

is singular by Proposition 3, where $\bar{\alpha}_{k}$ stands for the $k$-th element of the index subset $\bar{\alpha}$. From this fact, the same proposition, and Definition 7(ii), we observe that the matrix

$$
\left[\begin{array}{cc}
M_{\alpha \alpha} & p_{\alpha} \\
M_{\bar{\alpha}_{k} \alpha} & p_{\bar{\alpha}_{k}}
\end{array}\right]
$$

must be singular and the $k$-th element of the transform $\tilde{p}_{\bar{\alpha}}$ must be zero. Thus we have

$$
\left(M / M_{\alpha \alpha}\right)_{k k}=0 \Longrightarrow \tilde{p}_{\bar{\alpha}_{k}}=0 .
$$

(The argument is reversible so that the opposite direction of the above implication is also true.)

Condition (6) thus forces every pivot to be positive, and we may apply Algorithm 16. As mentioned earlier, the maximizing index $r$ cannot be chosen from the set $\alpha$. So it is clear in Step 3 that the cardinality of the set of basic $z$-variables increases by one with each iteration. It follows then that the algorithm must terminate with a solution function $z(\lambda)$ for all $\lambda \in \mathbb{R}$ in at $\operatorname{most} \max \left\{|\alpha|:\left(M_{\alpha \alpha}\right)^{-1}\right.$ exists $\} \leq \operatorname{rank}(M)$ iterations.

By setting $\lambda_{*}=0$ in Algorithm 16, it can be applied to solve the usual $\operatorname{LCP}(q, M)$. If we know that the conditions of the previous theorem are met by $M$, then a solution can be computed quickly. However, verifying those conditions may not be easy, and finding an efficient method to do so remains an open problem. 
Acknowledgements. The author would like to thank Professor Katta G. Murty for valuable guidance and feedback, and for suggesting the name "extended $n$-step." She is also grateful for the thoughtful comments provided by the anonymous referees, one of whom simplified the proof of Theorem 12.

\section{References}

1. Chandrasekaran, R.: A special case of the complementary pivot problem. Opsearch 7, 263-268 (1970)

2. Chu, T.H.: A class of strictly semimonotone matrices in linear complementarity theory. PhD thesis, University of Michigan, Ann Arbor, 2001

3. Chung, S.-J.: NP-Completeness of the linear complementarity problem. J. Optimiz. Theory. App. 60 (3), 393-399 (1989)

4. Cottle, R.W.: Monotone solutions of the parametric linear complementarity problem. Math. Program. 3, 210-224 (1972)

5. Cottle, R.W.: Completely-Q matrices. Math. Program. 19, 347-351 (1980)

6. Cottle, R.W., Dantzig, G.B.: Complementary pivot theory of mathematical programming. In: Dantzig, G.B., Veinott, Jr. A.F. (eds.), Mathematics of the Decision Sciences, Part 1, American Mathematical Society, Providence, Rhode Island, 1968, pp. 115-136

7. Cottle, R.W., Pang, J.-S., Stone, R.E.: The linear complementarity problem. Academic Press, Boston, 1992

8. Kelly, L.M., Murty, K.G., Watson, L.T.: CP-Rays in simplicial cones. Math. Program. 48, 387-414 (1990)

9. Kojima, M., Megiddo, N., Noma, T., Yoshise, A.: A unified approach to interior point algorithms for linear complementarity problems. In: Lecture Notes in Computer Science, Vol. 538, Springer-Verlag, New York, 1991

10. Lemke, C.E.: Bimatrix equilibrium points and mathematical programming. Manage. Sci. 11, 681-689 (1965)

11. Mohan, S.R.: On the simplex method and a class of linear complementarity problems. Linear Algebra Appl. 14, 1-9 (1976)

12. Murty, K.G.: Linear complementarity, linear and nonlinear programming. Heldermann Verlag, Berlin, 1988

13. Pang, J.-S.: Hidden Z-matrices with positive principal minors. Linear Algebra Appl. 23, 201-215 (1979)

14. Pang, J.-S., Chandrasekaran, R.: Linear complementarity problems solvable by a polynomially bounded pivoting algorithm. Math. Program. Study 25, 13-27 (1985)

15. Pang, J.-S., Lee, P. S-C.: A parametric linear complementarity technique for the computation of equilibrium prices in a single commodity spatial model. Math. Prog. 20, 81-102 (1981)

16. Reiman, M.I., Williams, R.J.: A boundary property of semimartingale reflecting Brownian motions. Prob. Theo. Related Fields 77, 87-97 (1988)

17. Saigal, R.: Lemke's algorithm and a special class of linear complementarity problems. Opsearch 8, 201208 (1971) 